Large-scale cosmic flows and moving dark energy

This article has been downloaded from IOPscience. Please scroll down to see the full text article.

JCAP03(2009)015

(http://iopscience.iop.org/1475-7516/2009/03/015)

View the table of contents for this issue, or go to the journal homepage for more

Download details:

IP Address: 147.96.14.15

The article was downloaded on 01/07/2013 at 15:48

Please note that terms and conditions apply. 


\title{
Large-scale cosmic flows and moving dark energy
}

\section{Jose Beltrán Jiménez and Antonio L. Maroto}

Departamento de Física Teórica I, Universidad Complutense de Madrid, 28040 Madrid, Spain

E-mail: jobeltra@fis.ucm.es, maroto@fis.ucm.es

Received November 28, 2008

Revised January 26, 2009

Accepted February 18, 2009

Published March 10, 2009

\begin{abstract}
Large-scale matter bulk flows with respect to the cosmic microwave background have very recently been detected on scales $\lesssim 100 h^{-1} \mathrm{Mpc}$ and $\lesssim 300 h^{-1} \mathrm{Mpc}$ by using two different techniques showing an excellent agreement in the motion direction. However, the unexpectedly large measured amplitudes are difficult to understand within the context of standard $\Lambda \mathrm{CDM}$ cosmology. In this work we show that the existence of such a flow could be signaling the presence of moving dark energy at the time when photons decoupled from matter. We also comment on the relation between the direction of the CMB dipole and the preferred axis observed in the quadrupole in this scenario.
\end{abstract}

Keywords: cosmic flows, dark energy theory 


\section{Contents}

1 Introduction 1

2 Moving dark energy $\quad 2$

3 CMB dipole from moving dark energy 3

4 Effects on the quadrupole $\quad 5$

5 Model example: scaling dark energy 6

$\begin{array}{lll}6 & \text { Discussion and conclusions } & 7\end{array}$

\section{Introduction}

Standard Model of cosmology describes an isotropic and homogeneous universe on very large scales which contains (dark and baryonic) matter, radiation and dark energy. However, at smaller scales, the universe is no longer homogeneous since matter density perturbations tend to form structures that originated by primordial inflationary fluctuations. Thus, small volumes of matter would acquire peculiar velocities with respect to the CMB rest frame. These peculiar velocities are due to the gravitational potential of outer structures and depend crucially on the matter power spectrum. In standard $\Lambda \mathrm{CDM}$, the large scale matter rest frame coincides with the CMB rest frame so that as we take larger and larger volumes of matter, peculiar velocities due to statistical fluctuations should become smaller and smaller. In fact, peculiar velocities are a very powerful tool in order to probe matter density fluctuations on very large scales and for that reason a great effort has been made in order to measure them by using different tracers: galaxies [1], clusters [2] or Type Ia supernovae [3]. Although on small scales $\left(\lesssim 60 h^{-1} \mathrm{Mpc}\right)$ surveys based on different distance indicators seem to agree with predictions of $\Lambda \mathrm{CDM}$ cosmology, on larger scales they have seemed to be in conflict among them for many years, yielding peculiar velocities in the wide range $0-1000 \mathrm{~km} / \mathrm{s}$. However, new data analysis performed in recent years [4] suggest that most of the surveys could also agree with each other on such large scales.

A completely new approach to obtain peculiar velocities of large volumes of matter which makes use of the Kinematic Sunyaev-Zeldovich effect on the CMB photons by the hot gas in clusters of galaxies was proposed in [5] and carried out very recently in [6]. They find coherent bulk flows on scales of $300 h^{-1} \mathrm{Mpc}$ towards $l=283^{\circ} \pm 14^{\circ}, b=11^{\circ} \pm 14^{\circ}$ (in galactic coordinates). The reported amplitude for those peculiar velocities are in the range $600-1000 \mathrm{~km} / \mathrm{s}$, although the authors point out that, even though there is no doubt about the existence and direction of the flows, the obtained values for the amplitudes may have some systematic offset. In [6], they attribute these peculiar velocities to pre-inflationary super-Hubble perturbations (see [7]).

Also, in a very recent work [8], a calculation of peculiar velocities using some of the available measurements has been performed, but with a new method which allows to reduce 
the sensitivity to small scale power and makes possible to compare the results obtained from different surveys. In that work, they find a consistent flow of matter on scales of $100 h^{-1} \mathrm{Mpc}$ towards $l=287^{\circ} \pm 9^{\circ}, b=8^{\circ} \pm 6^{\circ}$ and with an amplitude of $407 \pm 81 \mathrm{~km} / \mathrm{s}$. The direction of the detected flow is in very good agreement with the results in [6], in spite of having used a very different method. The authors of this work claim that these peculiar velocities may be due to structures larger than the reached scale of $100 h^{-1} \mathrm{Mpc}$.

In conclusion, these recent results suggest the existence of a coherent flow of matter with respect to the CMB rest frame on very large scales. In both works, such a flow is explained resorting to very large-scale matter perturbations, i.e., the observed peculiar velocities would be caused by the existence of some super-structure which must be further away than the Great Attractor, located at $40-60 h^{-1} \mathrm{Mpc}$ from us [9]. In any case, the presence of flows with such a large amplitude seems to be difficult to understand in the context of standard $\Lambda \mathrm{CDM}$ cosmology, which predicts much smaller velocities on the quoted scales.

In this work, we propose an alternative explanation in which the observed bulk flow could be indicating that matter is globally moving with respect to radiation, i.e., that matter and radiation do not share a common rest frame on very large scales. In other words, the velocity of a matter bulk of size $R$ may have two independent components: $\vec{V}_{R, b u l k}=\vec{V}_{R, s t a}+\vec{V}_{\text {cosmic }}$ where $\vec{V}_{R \text {,sta }}$ is the statistical r.m.s. velocity fluctuation generated by density inhomogeneities and $\vec{V}_{\text {cosmic }}$ is the cosmic velocity of matter with respect to the CMB because of having a different rest frame. Then, as we average over a large volume of matter, the first term becomes negligible whereas the second term remains constant and gives rise to a net cosmic flow. However, if that is the actual situation, then the question arises as how matter can be currently moving with respect to photons given the fact that they were strongly coupled in the early universe. In this work we propose that such velocity offset can be generated by the motion of dark energy with respect to the primordial plasma in the early universe.

\section{Moving dark energy}

In previous works $[10,11]$, the possibility of having dark energy with a different large-scale rest frame from that of the matter-radiation plasma in the early universe has been studied. If dark energy is a perfect fluid which has always been decoupled from matter and radiation, there is no reason to expect it to have the same rest frame as the other components of the universe. In such a case, the relative dark energy velocity $v_{\mathrm{DE}}$ should be considered as a free cosmological parameter, on equal footing with the density parameter $\Omega_{\mathrm{DE}}$ or equation of state $w_{\mathrm{DE}}$, and accordingly it could only be determined from observations. Indeed, the fact of having moving dark energy at the time when photons decoupled from matter allows to have relative velocities between dark matter, baryons and photons. This relative motion may be precisely the one detected in $[6,8]$.

The model proposed in [10, 11] consists of a universe filled with four perfect fluids: baryons, radiation, dark matter and dark energy, whose velocities can differ from each other. In that case, we can define the velocity of the Cosmic Center of Mass (CCM), for small velocities, as:

$$
\vec{S}=\frac{\sum_{\alpha}\left(\rho_{\alpha}+p_{\alpha}\right) \vec{v}_{\alpha}}{\sum_{\alpha}\left(\rho_{\alpha}+p_{\alpha}\right)}
$$


where $\alpha$ stands for the four aforementioned components of the universe. Now, if we place ourselves in the CCM rest frame, defined by the condition $\vec{S}=0$, the following relation must hold:

$$
\sum_{\alpha}\left(\rho_{\alpha}+p_{\alpha}\right) \vec{v}_{\alpha}=0
$$

In the early universe, radiation dominates over the rest of components so it will drag baryons and dark matter particles in such a way that they all will share a common rest frame, as expected for interacting species in thermal equilibrium. Concerning dark energy, since it does not interact with photons, it can move in a different way. However, as long as we use the CCM rest frame, photons and dark energy velocities are related by means of (2.2) as follows:

$$
\vec{v}_{\mathrm{DE}}^{\text {early }}=-\frac{4}{3} \frac{\rho_{R}^{\text {early }}}{\rho_{\mathrm{DE}}^{\text {early }}+p_{\mathrm{DE}}^{\text {early }}} \vec{v}_{R}^{\text {early }}
$$

where we have used that matter and radiation velocities are the same and that matter energy density is negligible compared to that of radiation. Notice that this relation provides a preferred axis in the universe given by the fluids motion direction. In other words, the universe will have axial symmetry around the axis defined by the motion of the different components.

On the other hand, the momentum conservation equation for each fluid reads:

$$
\frac{d}{d t}\left[a^{4}\left(\rho_{\alpha}+p_{\alpha}\right)\left(\vec{S}-\vec{v}_{\alpha}\right)\right]=0
$$

which can be immediately integrated assuming constant equation of state $w_{\alpha}=\frac{p_{\alpha}}{\rho_{\alpha}}$ to give:

$$
\vec{S}-\vec{v}_{\alpha}=\vec{v}_{\alpha}^{0} a^{3 w_{\alpha}-1}
$$

with $\vec{v}_{\alpha}^{0}$ the present value of the velocity. Thus, radiation $\left(w_{R}=\frac{1}{3}\right)$ moves with constant velocity with respect to the CCM, whereas matter $(w=0)$ velocity decays as $a^{-1}$. The evolution of dark energy velocity depends on the particular model under consideration. Therefore, according to (2.5), matter and radiation have constant velocity before decoupling, but, after that, matter starts reducing its velocity as the universe expands whereas radiation keeps moving with the same constant velocity. As a consequence, the presence of moving dark energy at the time of recombination makes possible that matter and radiation could acquire a relative velocity after decoupling. This effect can only take place if dark energy is not a cosmological constant, otherwise there would be no momentum contribution from dark energy to (2.2). Notice that, since dark matter particles should decouple before baryons do (in order to be able to form the structures we observe today) and they both have the same equation of state, we expect them to have a relative motion with constant velocity. Thus, the complete picture of the motions would be as follows: radiation moving with constant velocity, baryons and dark matter moving in the same direction with constant relative velocity and, both, slowing down with respect to photons. Finally, dark energy would move along the opposite direction.

\section{CMB dipole from moving dark energy}

In the CCM rest frame, the metric becomes diagonal and can be written as that of an axisymmetric Bianchi I space-time. Moreover, as the velocities of the fluids are small, this metric can be treated as a Robertson-Walker metric with a small perturbation. Thus, the 
usual expression for the dipole given by the Sachs-Wolfe effect at first order applies to this case, although we have to read the velocities as relative to the CCM. Hence, the dipole in an arbitrary frame for this model is given by [10]:

$$
\frac{\delta T_{\text {dipole }}}{T_{0}} \simeq \vec{n} \cdot(\vec{S}-\vec{v})_{\mathrm{dec}}^{0}
$$

where $\vec{n}$ is a unitary vector along the direction of observation, $\vec{v}_{0}$ is the velocity of the observer today and $\vec{v}_{\text {dec }}$ is the velocity of the emitter at decoupling time. Notice that according to (3.1) when several fluids with relative velocities are present, the CMB dipole is given by the velocities of emitter and observer with respect to the CCM frame. In the case in which all the fluids share a common rest frame, the velocites are referred to that frame which is nothing but the CMB frame. However, when the fluids velocities are different, the physically relevant frame for the dipole is the CCM which is different from the CMB frame. In this sense, if emission took place from a source at rest with respect to the CCM frame, that frame could be determined physically as the frame attached to an observer who measures a vanishing dipole.

If we assume that the intrinsic dipole fluctuation at the last scattering surface is negligible we can take $\vec{v}_{\text {dec }} \simeq \vec{v}_{R}^{\text {dec }}$. On the other hand, if the observer today is at rest with respect to matter we have $\vec{v}_{0} \simeq \vec{v}_{M}^{0}$. Thus, if we now refer the velocities to the CCM frame, we finally get:

$$
\frac{\delta T_{\text {dipole }}}{T_{0}} \simeq \vec{n} \cdot\left(\vec{v}_{R}^{0}-\vec{v}_{M}^{0}\right)
$$

where we have used that the velocity of radiation with respect to the CCM is constant. Then, according to (3.2), we conclude that the contribution to the dipole temperature fluctuation of the CMB is due to the relative motion of matter with respect to radiation. This is precisely the kind of flow detected in $[6,8]$ so the direction reported in both papers gives directly the direction of motion of the fluids.

Notice that, although calculated in the CCM frame, (3.2) is valid for any frame since it is expressed as the difference of two velocities evaluated at the same time. According to (2.5), today the velocity of matter with respect to the CCM is expected to be much smaller than that of radiation and the dipole can also be written as:

$$
\frac{\delta T_{\text {dipole }}}{T_{0}} \simeq \vec{n} \cdot \vec{v}_{R}^{0}
$$

so that the cosmological dipole could be alternatively interpreted as due to the relative motion of radiation with respect to the CCM.

Using again expression (2.2), it is possible to relate the amplitude of the dipole $\left(v_{R}^{0}\right)$ to the present value of the dark energy velocity with respect to the CCM frame:

$$
v_{\mathrm{DE}}^{0}=\frac{v_{R}^{0}}{\left(1+w_{\mathrm{DE}}^{0}\right) \Omega_{\mathrm{DE}}}\left(\frac{2}{3} \Omega_{R}+\frac{\Omega_{B}}{1+z_{\mathrm{dec}}}+\frac{\Omega_{\mathrm{DM}}}{1+z_{*}}\right)
$$

where $z_{*}$ is the decoupling redshift of dark matter and radiation and $w_{\mathrm{DE}}^{0}$ is the present value of the dark energy equation of state. We can estimate typical values of the present dark energy velocity. Thus, from the measured bulk flows we can take $v_{R}^{0} \sim 500 \mathrm{~km} / \mathrm{s}$ and assuming $\Omega_{\mathrm{DE}} \simeq 0.7, z_{*}>10^{5}$ and $w_{\mathrm{DE}}^{0} \simeq-0.97$, we get $v_{\mathrm{DE}}^{0} \sim 1 \mathrm{~km} / \mathrm{s}$. 


\section{Effects on the quadrupole}

The relative motion of the different components in the universe also affects higher multipoles of the CMB. In general, the contribution to the $\ell$ th multipole will be $\sim|\vec{v}|^{\ell}$ so the effect decreases rapidly as we increase $\ell$ because of the smallness of the velocities.

In particular, for quadrupolar temperature fluctuation it is possible to obtain [11]:

$$
\frac{\delta T_{Q}}{T_{0}}=-\frac{1}{2} h\left(\hat{v}_{i} \hat{v}_{j}-\frac{1}{3} \delta_{i j}\right) n^{i} n^{j},
$$

where $h$ is a function which measures the variation of the degree of anisotropy from the last scattering surface until present and depends on the particular dark energy model under consideration and $\hat{v}_{i}$ is a unitary vector pointing along the direction of the motion of the fluids. Moreover, if such a direction is given by $(\theta, \phi)$, the components of the quadrupole are:

$$
\begin{aligned}
& a_{20}^{A}=\frac{\sqrt{\pi}}{6 \sqrt{5}} h[1+3 \cos 2 \theta], \\
& a_{21}^{A}=-\left(a_{2-1}^{A}\right)^{*}=-\sqrt{\frac{\pi}{30}} h e^{-i \phi} \sin 2 \theta, \\
& a_{22}^{A}=\left(a_{2-2}^{A}\right)^{*}=\sqrt{\frac{\pi}{30}} h e^{-2 i \phi} \sin ^{2} \theta .
\end{aligned}
$$

Then, the quadrupole generated by the motion is:

$$
Q_{A} \equiv \sqrt{\frac{3}{5 \pi} \sum_{m=-2}^{2}\left|a_{2 m}^{A}\right|^{2}}=\frac{2}{5 \sqrt{3}} h .
$$

On the other hand, we still have to add the isotropic quadrupole $Q_{I}$ coming from the inflationary fluctuations, whose components can be written as:

$$
\begin{aligned}
& a_{20}^{I}=\sqrt{\frac{\pi}{3}} e^{i \alpha_{1}} Q_{I}, \\
& a_{21}^{I}=-\left(a_{2-1}^{I}\right)^{*}=\sqrt{\frac{\pi}{3}} e^{i \alpha_{2}} Q_{I}, \\
& a_{22}^{I}=\left(a_{2-2}^{I}\right)^{*}=\sqrt{\frac{\pi}{3}} e^{i \alpha_{3}} Q_{I},
\end{aligned}
$$

where the phase factors $\alpha_{i}$ can be considered as stochastic variables. As the anisotropy is expected to be small, the total effect is given by the linear superposition of both contributions [12], i.e., $a_{\ell m}^{T}=a_{\ell m}^{A}+a_{\ell m}^{I}$. Now, in a frame with the $z$-axis parallel to $\hat{v}_{i}$ we have $\theta=0$ so that the only non-vanishing anisotropic component is $a_{20}^{A}$. That way, the only modified component of the total quadrupole, in that frame, is $a_{20}$. However, in a general frame with the velocities along the direction $(\theta, \phi)$ the total quadrupole becomes [13]:

$$
Q_{T}^{2}=Q_{A}^{2}+Q_{I}^{2}-2 f Q_{A} Q_{I}
$$

where

$$
\begin{aligned}
f=\frac{1}{4 \sqrt{5}} & {\left[2 \sqrt { 6 } \left[-\sin \theta \cos \left(2 \phi+\alpha_{3}\right)\right.\right.} \\
& \left.\left.+2 \cos \theta \cos \left(\phi+\alpha_{2}\right)\right] \sin \theta-(1+3 \cos (2 \theta)) \cos \alpha_{1}\right] .
\end{aligned}
$$


Assuming that the anisotropic quadrupole is a new contribution to add on top of the true model which provides the observed quadrupole, it has to satisfy [11]:

$$
\begin{aligned}
& 0 \mu \mathrm{K}^{2} \lesssim\left(\delta T_{A}\right)^{2} \lesssim 1861 \mu \mathrm{K}^{2} \quad 68 \% \text { C.L. } \\
& 0 \mu \mathrm{K}^{2} \lesssim\left(\delta T_{A}\right)^{2} \lesssim 5909 \mu \mathrm{K}^{2} \quad 95 \% \text { C.L. }
\end{aligned}
$$

in order not to have a too large total quadrupole. These conditions yield the following constraints on the parameter $h$ :

$$
\begin{aligned}
& 0 \lesssim h \lesssim 6.92 \times 10^{-5} \quad 68 \% \text { C.L. } \\
& 0 \lesssim h \lesssim 1.23 \times 10^{-4} \quad 95 \% \text { C.L. }
\end{aligned}
$$

\section{Model example: scaling dark energy}

Scaling models [14] are those with equation of state such that dark energy mimics the dominant component of the universe throughout most of the universe evolution. Thus, dark energy evolves as radiation before matter-radiation equality and as matter after that. However, in order to explain the accelerated expansion of the universe, dark energy has to exit from that regime and join into one with $w_{\mathrm{DE}}<-1 / 3$ at some point. Then, the evolution of the dark energy density is given by:

$$
\rho_{\mathrm{DE}}=\left\{\begin{array}{lr}
\rho_{\mathrm{DE}}^{0} a_{T}^{-3 w_{\mathrm{DE}}} a_{\mathrm{eq}} a^{-4} & a<a_{\mathrm{eq}} \\
\rho_{\mathrm{DE}}^{0} a_{T}^{-3 w_{\mathrm{DE}}} a^{-3} & a_{\mathrm{eq}}<a<a_{T} \\
\rho_{\mathrm{DE}}^{0} a^{-3\left(w_{\mathrm{DE}}+1\right)} & a>a_{T}
\end{array}\right.
$$

where as commented before, $a_{T}$ is the scale factor when dark energy leaves the scaling regime and $\rho_{\mathrm{DE}}^{0}$ is the present value of the dark energy density.

In the evolution of dark energy velocity, we have to take into account the momentum conservation equation given by (2.4). This equation implies that the dark energy velocity must be discontinuous at the transition points since the equation of state jumps at those times whereas the quantity $a^{4}\left(1+w_{\mathrm{DE}}\right) \rho_{\mathrm{DE}} \vec{v}_{\mathrm{DE}}$ is constant, being $\rho_{\mathrm{DE}}$ continuous. With this in mind, we get the following evolution for dark energy velocity in the CCM frame:

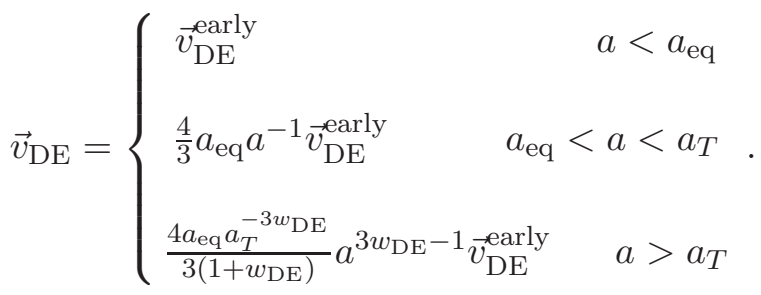

In figure 1, we see the typical behavior of fluids velocities. In these models, the quadrupole can be approximated by (see [11]) $Q_{A} \simeq 0.44 \epsilon\left(\vec{v}_{\mathrm{DE}}^{\text {early }}\right)^{2}$ where $\vec{v}_{\mathrm{DE}}^{\text {early }}$ relates to radiation velocity through $(2.3)$ as follows: $v_{R}=\epsilon v_{\mathrm{DE}}^{\text {early }}$ being $\epsilon$ the initial fraction of dark energy density. With this relation we can express the quadrupole as $Q_{A} \simeq 0.44 v_{R}^{2} \epsilon^{-1} \simeq$ $1.23 \times 10^{-6} \epsilon^{-1}$ or, equivalently, we have that $h \simeq 5.32 \times 10^{-6} \epsilon^{-1}$, where we have assumed 


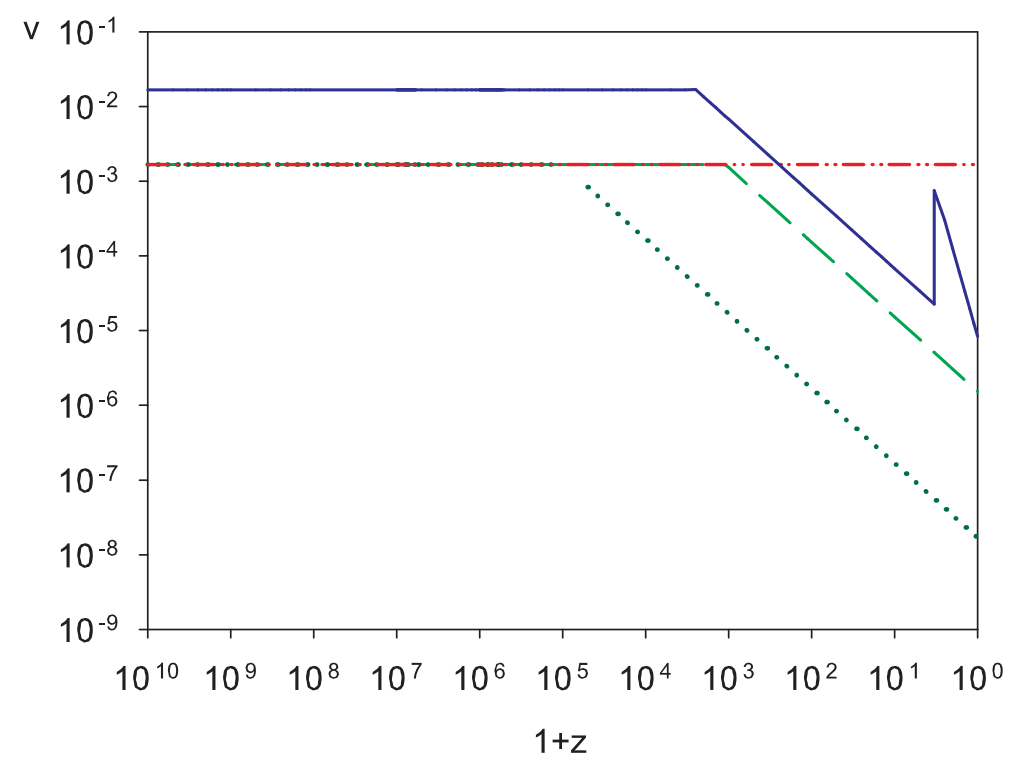

Figure 1. Velocities evolution (CCM frame) in a scaling model with $v_{R}=1.67 \times 10^{-3} \mathrm{c}$ and $\epsilon=0.1$. The continuous line (blue) is for dark energy, dashed-dotted (red) for radiation, dotted (cyan) for dark matter and dashed (green) for baryonic matter. Notice that in this plot dark matter is assumed to decouple at $z_{*} \simeq 10^{5}$. [11]

that the total bulk flow is due to the motion of the fluids and we have taken the velocity of radiation to be $v_{R} \simeq 500 \mathrm{~km} / \mathrm{s}=1.67 \times 10^{-3} \mathrm{c}$ in the CCM rest frame. Therefore, constraints (4.8) read:

$$
\begin{array}{lll}
7.69 \times 10^{-2} & \lesssim \epsilon \lesssim 0.2 & 68 \% \text { C.L. } \\
4.32 \times 10^{-2} \lesssim \epsilon \lesssim 0.2 & 95 \% \text { C.L. }
\end{array}
$$

where the upper limit comes from primordial nucleosynthesis, which imposes the amount of dark energy density at that time to be less than about $20 \%$ [15] so that $\epsilon \lesssim 0.2$.

To summarize, in this kind of models it is possible to explain the presence of the matter bulk flow from the dark energy motion in a compatible way with the measurements of the CMB quadrupole.

\section{Discussion and conclusions}

Finally, we would like to comment on the fact that the total quadrupole has a preferred axis which happens to coincide with the direction of the velocities and, as a consequence, with that of the dipole. Therefore, a moving dark energy model could also shed some light on the so-called axis of evil problem [16]. Although this anomaly usually refers to the observed alignment of the $\ell=2-5$ multipoles, there are also evidence that the axis of such alignment is correlated with the dipole direction at more than 99\% C.L. [17]. Since moving dark energy gives a common physical mechanism for both the dipole and quadrupole contributions, it is expected to have correlations among them. Indeed, in [11] it is shown that the motion of dark energy could solve the low quadrupole anomaly for some models of dark energy, in particular, for scaling and null dark energy models. The solution of the low quadrupole problem arises 


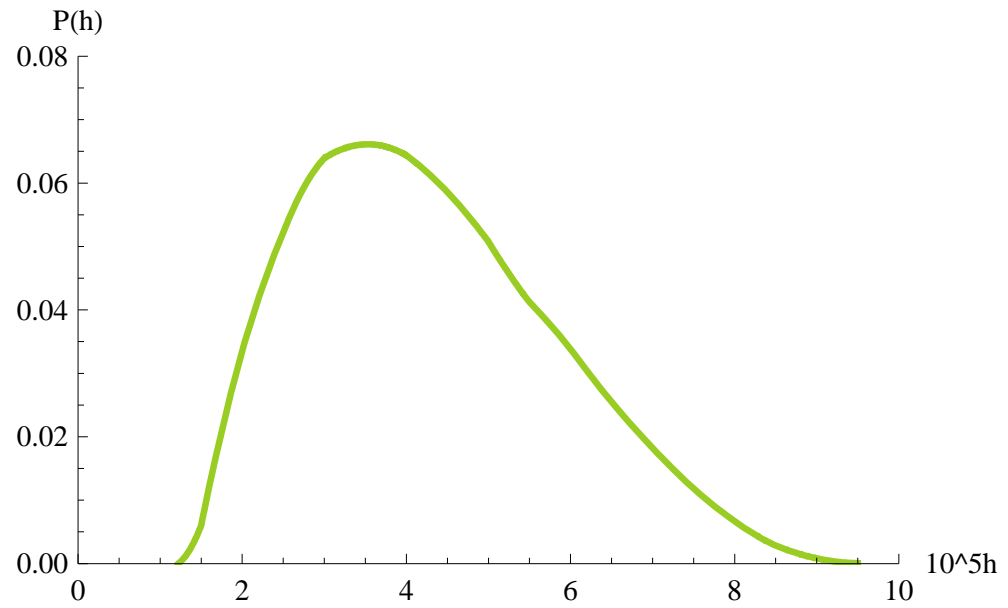

Figure 2. Probability of having a quadrupole within $1 \sigma$ interval of the measurements as a function of the parameter $h$, which contains the information about the dark energy model.

because the relative motion of the fluids generates a certain degree of anisotropy which is seen by the photons coming from the last scattering surface and acquire a quadrupolar anisotropy. In a frame with the $z$-axis pointing along the fluids motions, the power of the quadrupole given by the generated anisotropy is zonal, i.e., it is concentrated in the $m=0$ component so that it gives rise to a cylindrical contribution. However, we still have to add the standard isotropic fluctuation generated during inflation, whose components are all comparable. Then, if we add linearly both contributions in such a way that the resulting quadrupole is lower than the inflationary one, the suppression has to take place for the $m=0$ component and, therefore, the total quadrupole will be non-cylindrical.

Thus, if we want to explain the low quadrupole with this new contribution plus the standard inflation contribution, we need [11]:

$$
\begin{aligned}
& 54 \mu \mathrm{K}^{2} \lesssim\left(\delta T_{A}\right)^{2} \lesssim 3857 \mu \mathrm{K}^{2} \quad 68 \% \text { C.L. } \\
& 0 \mu \mathrm{K}^{2} \lesssim\left(\delta T_{A}\right)^{2} \lesssim 9256 \mu \mathrm{K}^{2} \quad 95 \% \text { C.L. }
\end{aligned}
$$

which leads to the following constraints on $h$ :

$$
\begin{aligned}
1.18 \times 10^{-5} & \lesssim h \lesssim 9.96 \times 10^{-5} \quad 68 \% \text { C.L. } \\
0 & \lesssim h \lesssim 1.54 \times 10^{-4} \quad 95 \% \text { C.L. }
\end{aligned}
$$

For scaling models, we have that the low quadrupole can be explained if the following constraints hold:

$$
\begin{array}{lll}
5.34 \times 10^{-2} & \lesssim \epsilon \lesssim 0.2 \quad 68 \% \text { C.L. } \\
3.45 \times 10^{-2} & \lesssim \epsilon \lesssim 0.2 \quad 95 \% \text { C.L. }
\end{array}
$$

As the initial conditions are supposed to be random, we can compute the likelihood of explaining the low quadrupole with the anisotropic contribution as a function of the modeldependent parameter $h$. To that end, we perform simulations of the total quadrupole by choosing the phases $\alpha_{i}$ and the direction $(\theta, \phi)$ randomly. In figure 2 we can see that the 
maximum of the likelihood happens for $h \simeq 3.5 \times 10^{-5}$ for which the chance of having a quadrupole as low as the observed one is $\simeq 7 \%$.

In conclusion, we have shown that the detected large scale matter bulk flow could be signalling the presence of a dark energy flow which would be responsible for the present relative motion of matter and radiation. This kind of explanation could also shed some light on the problem of the low quadrupole and the alignment of dipole and quadrupole axes.

\section{Acknowledgments}

We would like to thank Fernando Atrio Barandela for useful discussions and suggestions. This work has been supported by DGICYT (Spain) project numbers FPA 2004-02602 and FPA 2005-02327, UCM-Santander PR34/07-15875, CAM/UCM 910309 and MEC grant BES2006-12059.

\section{References}

[1] A. Dressler et al., Spectroscopy and photometry of elliptical galaxies. 1. A new distance estimator, Astrophys. J. 313 (1987) 42 [SPIRES];

D.S. Mathewson, V.L. Ford and M. Buchhorn, No back-side infall into the great attractor, Astrophys. J. 389 (1992) L5;

J.A. Willick, The Las Campanas observatory/Palomar 10,000 kilometer per second cluster survey. II. Constraints on large-scale streaming, Astrophys. J. 522 (1999) 647;

S. Courteau et al., Shellflow. I. The convergence of the velocity field at 6000 kilometers per second, Astrophys. J. 544 (2000) 636 [astro-ph/0002420] [SPIRES].

[2] T.R. Lauer and M. Postman, The motion of the local group with respect to the 15, 000 kilometer per second Abell cluster inertial frame, Astrophys. J. 425 (1994) 418;

M.J. Hudson and H. Ebeling, The environmental dependence of brightest cluster galaxies: implications for large-scale flows, Astrophys. J. 479 (1997) 621 [astro-ph/9611083] [SPIRES]; M.J. Hudson, R.J. Smith, J.R. Lucey, D.J. Schlegel and R.L. Davies, A large-scale bulk flow of galaxy clusters, Astrophys. J. 512 (1999) L79 [astro-ph/9901001] [SPIRES].

[3] A. Riess et al., The velocity field from type IA supernovae matches the gravity field from galaxy surveys, Astrophys. J. 488 (1997) L1 [astro-ph/9707261] [SPIRES].

[4] R. Juszkiewicz, et al., Evidence for a low-density universe from the relative velocities of galaxies, Science $\mathbf{2 8 7}$ (2000) 109 [astro-ph/0001041] [SPIRES];

H.A. Feldman, R. Watkins, A.L. Melott and S.W. Chambers, Optimal moments for the analysis of peculiar velocity surveys II: testing, Astrophys. J. 599 (2003) 820 [astro-ph/0304316] [SPIRES];

D.J. Radburn-Smith, J.R. Lucey and M.J. Hudson, A comparison of local type Ia supernovae with the IRAS PSCz gravity field, Mon. Not. Roy. Astron. Soc. 355 (2004) 1378;

R.W. Pike and M.J. Hudson, Cosmological parameters from the comparison of the $2 M A S S$ gravity field with peculiar velocity surveys, Astrophys. J. 635 (2005) 11 [astro-ph/0511012] [SPIRES];

D. Sarkar, H.A. Feldman and R. Watkins, Bulk flows from velocity field surveys: a consistency check, Mon. Not. Roy. Astron. Soc. 375 (2007) 691 [astro-ph/0607426] [SPIRES];

R. Watkins and H.A. Feldman, Power spectrum shape from peculiar velocity data, Mon. Not. Roy. Astron. Soc. 379 (2007) 343 [astro-ph/0702751] [SPIRES]; Bulk flow and shear moments of the SFI++ survey, Mon. Not. Roy. Astron. Soc. 387 (2008) 825 [arXiv:0802.2961] [SPIRES]. 
[5] A. Kashlinsky and F. Atrio-Barandela, Measuring cosmological bulk flows via the kinematic Sunyaev-Zeldovich effect in the upcoming cosmic microwave background maps, Astrophys. J. 536 (2000) L67 [astro-ph/0005197] [SPIRES].

[6] A. Kashlinsky, F. Atrio-Barandela, D. Kocevski and H. Ebeling, A measurement of large-scale peculiar velocities of clusters of galaxies: technical details, Astrophys. J. 691 (2009) 1479 [arXiv:0809.3733] [SPIRES]; A measurement of large-scale peculiar velocities of clusters of galaxies: results and cosmological implications, arXiv:0809.3734 [SPIRES].

[7] M.S. Turner, A tilted universe (and other remnants of the preinflationary universe), Phys. Rev. D 44 (1991) 3737 [SPIRES].

[8] R. Watkins, H.A. Feldman and M.J. Hudson, Consistently large cosmic flows on scales of $100 \mathrm{Mpc} / \mathrm{h}$ : a challenge for the standard $\Lambda$ CDM cosmology, arXiv:0809.4041 [SPIRES].

[9] D. Lynden-Bell et al., Spectroscopy and photometry of elliptical galaxies. V - Galaxy streaming toward the new supergalactic center, Astrophys. J. 326 (1988) 19 [SPIRES]; A. Dresler, The supergalactic plane redshift survey - a candidate for the great attractor, Astrophys. J. 329 (1988) 519.

[10] A.L. Maroto, Moving dark energy and the CMB dipole, JCAP 05 (2006) 015 [astro-ph/0512464] [SPIRES]; Dark energy in motion, Int. J. Mod. Phys. D 15 (2006) 2165 [astro-ph/0605381] [SPIRES]; Dark energy rest frame and the CMB dipole, AIP Conf. Proc. 878 (2006) 240 [astro-ph/0609218] [SPIRES];

[11] J. Beltran Jimenez and A.L. Maroto, Cosmology with moving dark energy and the CMB quadrupole, Phys. Rev. D 76 (2007) 023003 [astro-ph/0703483] [SPIRES].

[12] E.F. Bunn, P. Ferreira and J. Silk, How anisotropic is our universe?, Phys. Rev. Lett. 77 (1996) 2883 [astro-ph/9605123] [SPIRES].

[13] L. Campanelli, P. Cea and L. Tedesco, Ellipsoidal universe can solve the CMB quadrupole problem, Phys. Rev. Lett. 97 (2006) 131302 [Erratum ibid. 97 (2006) 209903] [astro-ph/0606266] [SPIRES].

[14] S. Capozziello, A. Melchiorri and A. Schirone, Scaling dark energy, Phys. Rev. D 70 (2004) 101301 [astro-ph/0408112] [SPIRES];

E.J. Copeland, A.R. Liddle and D. Wands, Exponential potentials and cosmological scaling solutions, Phys. Rev. D 57 (1998) 4686 [gr-qc/9711068] [SPIRES];

P.G. Ferreira and M. Joyce, Cosmology with a primordial scaling field, Phys. Rev. D 58 (1998) 023503 [astro-ph/9711102] [SPIRES].

[15] E.W. Kolb and M.S. Turner, The early universe, Addison-Wesley, U.S.A. (1990).

[16] K. Land and J. Magueijo, The axis of evil, Phys. Rev. Lett. 95 (2005) 071301 [astro-ph/0502237] [SPIRES];

K. Land and J. Magueijo, The axis of evil revisited, Mon. Not. Roy. Astron. Soc. 378 (2007) 153 [astro-ph/0611518] [SPIRES].

[17] D.J. Schwarz, G.D. Starkman, D. Huterer and C.J. Copi, Is the low-l microwave background cosmic?, Phys. Rev. Lett. 93 (2004) 221301 [astro-ph/0403353] [SPIRES];

C. Copi, D. Huterer, D. Schwarz and G. Starkman, The Uncorrelated Universe: statistical Anisotropy and the Vanishing Angular Correlation Function in WMAP Years 1-3, Phys. Rev. D 75 (2007) 023507 [astro-ph/0605135] [SPIRES]. 